PROCEEDINGS OF THE

AMERICAN MATHEMATICAL SOCIETY

Volume 136, Number 8, August 2008, Pages 2665-2674

S 0002-9939(08)09256-3

Article electronically published on March 27, 2008

\title{
SOLUTION OF THE KARLIN PROBLEM FOR ZERO-DIMINISHING SEQUENCES SATISFYING A CARLEMAN CONDITION
}

\author{
ANDREW BAKAN AND STEPHAN RUSCHEWEYH
}

(Communicated by David Preiss)

\begin{abstract}
We describe all zero-diminishing sequences (over the real-valued polynomials on $\mathbb{R}$ ) which additionally satisfy a Carleman condition and show that they are of the same kind as those in E. Laguerre's theorem from 1884.
\end{abstract}

\section{INTRODUCTION AND MAIN RESULTS}

Denote by $\mathcal{P}$ the set of all real algebraic polynomials. A sequence of real numbers $\gamma:=\left\{\gamma_{n}\right\}_{n \geq 0}$ is called a zero-diminishing sequence if for any polynomial $p(x)=$ $p_{0}+p_{1} x+\ldots+p_{m} x^{m}$ with real coefficients (i.e. $p \in \mathcal{P}$ ) the number of real zeros of the transformed polynomial $T_{\gamma} p$ with $T_{\gamma} p(x):=\gamma_{0} p_{0}+\gamma_{1} p_{1} x+\ldots+\gamma_{m} p_{m} x^{m}$ is not greater than the number of real roots of $p$. In other words,

$$
\mathbb{Z}_{\mathbb{R}}\left(T_{\gamma} p\right) \leq \mathbb{Z}_{\mathbb{R}}(p),
$$

where $\mathbb{Z}_{A}(p)$ denotes the number of zeros of the non-constant polynomial $p$ in the set $A \subset \mathbb{C}$, counting multiplicities, while $\mathbb{Z}_{A}(a)=0$ for every real number $a$. Let $\mathcal{Z D}$ denote the set of all zero-diminishing sequences in the sense of (1.1) and which are not of the form $\left\{\gamma_{0}, 0, \ldots, 0, \ldots\right\}$.

Let $\mathcal{L P}_{2}^{+}$denote the set of Laguerre-Pólya functions, i.e. the entire functions with a representation

$$
e^{-a x^{2}+b x} \prod_{k=1}^{m}\left(1+\frac{x}{\alpha_{k}}\right) e^{-\frac{x}{\alpha_{k}}}
$$

where $m \in \mathbb{N} \cup\{\infty\}$ and

$$
a, b, \alpha_{k} \in \mathbb{R} \text {, with } a \geq 0, \alpha_{k}>0 \text { for all } k=1, \ldots, m \text {, and } \sum_{k=1}^{m} \frac{1}{\alpha_{k}^{2}}<\infty ;
$$

compare [18, p. 8] or [17, Ch. III, §3]. In 1884, E. Laguerre [21, p. 116] proved that for every entire function $\Phi \in \mathcal{L P}_{2}^{+}$we have $\{1 / \Phi(n)\}_{n \geq 0} \in \mathcal{Z D}$. We say

Received by the editors October 28, 2005, and, in revised form, April 10, 2007.

2000 Mathematics Subject Classification. Primary 12D10, 26C10, 30E05; Secondary 30C15, $30 \mathrm{D} 15$.

Key words and phrases. Zero-diminishing sequences, moment problems, zeros of polynomials.

This work was completed while A. Bakan was visiting Würzburg University, supported by the German Academic Exchange Service (DAAD, grant 322-A/02/12977). S. Ruscheweyh acknowledges partial support from the German-Israeli Foundation (grant G-809-234.6/2003).

(C)2008 American Mathematical Society Reverts to public domain 28 years from publication 
that a sequence $\gamma \in \mathcal{Z D}$ is of Laguerre type if $\gamma_{n}=\gamma_{0} / \Phi(n), n \geq 0$, for some $\Phi \in \mathcal{L P}_{2}^{+}, \gamma_{0}>0$.

This theorem of Laguerre led S. Karlin [20, p. 382] to the following question (which has ever since been referred to as the Karlin problem): are there zerodiminishing sequences which are (essentially) of non-Laguerre type? Here the word "essential" hints at the fact that $\gamma:=\left\{\gamma_{n}\right\}_{n \geq 0} \in \mathcal{Z D}$ implies

$$
c \gamma:=\left\{c \gamma_{n}\right\}_{n \geq 0} \in \mathcal{Z D} \text { for } c \neq 0 \text { and } \gamma^{*}:=\left\{(-1)^{n} \gamma_{n}\right\}_{n \geq 0} \in \mathcal{Z D} \text {. }
$$

Karlin's problem seems to be still open 1

In 1996, A. Bakan, T. Craven, G. Csordas and A. Golub [3] took up this problem and proved in [3, Lem. 3] that if one (and therefore all) of the real sequences $\gamma,-\gamma, \gamma^{*},-\gamma^{*}$ belongs to $\mathcal{Z D}$, then (exactly) one of them has only positive entries (like the ones of Laguerre type). Therefore it will be sufficient to study positive zero-diminishing sequences. The set of those sequences will be denoted by $\mathcal{Z D}^{+}$.

With every $\gamma \in \mathcal{Z D}^{+}$we associate the formal power series

$$
\Gamma_{\gamma}(z):=\sum_{n=0}^{\infty} \gamma_{n} z^{n}
$$

It was proved in [5, Lem. 1] that for every $\gamma \in \mathcal{Z D}^{+}$there exists a non-negative Borel measure $\mu_{\gamma}$ on $\mathbb{R}^{+}:=[0,+\infty)$ with all moments finite such that

$$
\gamma_{n}=\int_{0}^{\infty} t^{n} d \mu_{\gamma}(t), \quad n \geq 0 .
$$

Representation (1.3) and the Cauchy-Schwarz inequality allow us to estimate the second-order Hankel determinants (see [28, Th. 1.2]) of the shifted zero-diminishing sequence $\left\{\gamma_{n+m}\right\}_{n \geq 0}$ with $m \geq \mathrm{d}^{2}$ to get the inverse Turan inequalities for $\gamma \in \mathcal{Z D}^{+}$ (see [13):

$$
\left|\begin{array}{cc}
\gamma_{n} & \gamma_{n+1} \\
\gamma_{n+1} & \gamma_{n+2}
\end{array}\right|=\gamma_{n} \gamma_{n+2}-\gamma_{n+1}^{2} \geq 0, \quad n \geq 0
$$

This implies, in particular, that

$$
0 \leq \lim _{n \rightarrow \infty} \frac{\gamma_{n}}{\gamma_{n+1}}=\lim _{n \rightarrow \infty} \gamma_{n}^{-1 / n}<\infty
$$

see [26, 3.37]. Theorem A below solves completely the cases in (1.5) with $Q(\gamma):=$ $\lim _{n \rightarrow \infty} \gamma_{n}{ }^{-1 / n}>0$, i.e. if $\Gamma_{\gamma}(z)$ has a positive radius of convergence.

Theorem A $([3,1996)$. For any sequence $\gamma$ of positive real numbers with $Q:=$ $Q(\gamma)>0$ the following statements are equivalent:

(a) $\gamma \in \mathcal{Z D}^{+}$.

(b) There exists $\Phi \in \mathcal{L P}_{1}(\log Q)$ such that $\gamma_{n}=\frac{\gamma_{0}}{\Phi(n)}, n \geq 0$.

(c) For all $A>0$ and $p \in \mathcal{P}$ we have $\mathbb{Z}_{[0, A \cdot Q]}\left(T_{\gamma} p\right) \leq \mathbb{Z}_{[0, A]}(p)$.

Here

$$
\mathcal{L P}_{1}(a):=\left\{e^{a x} \prod_{k \geq 1}\left(1+\frac{x}{\alpha_{k}}\right) \mid \alpha_{k}>0 \text { for } k \geq 1 \text {, and } \sum_{k \geq 1} \frac{1}{\alpha_{k}}<\infty\right\} .
$$

In particular Theorem A implies:

\footnotetext{
${ }^{1}$ It was shown in [5] and [15 that the solution proposed in [14] is incorrect.

${ }^{2}$ It follows from (1.1) applied to $x^{m} p(x), p \in \mathcal{P}$, that $\left\{\gamma_{n+m}\right\}_{n \geq 0} \in \mathcal{Z D}^{+}$for all $m \geq 0$.
} 
Corollary A. Let $\gamma \in \mathcal{Z D}^{+}$be such that $\Gamma_{\gamma}$ has a positive radius of convergence. Then $\gamma$ is of Laguerre type.

In view of (1.3) Karlin's problem can be reformulated as follows: for which measures on $[0,+\infty)$ do the moments form a zero-diminishing sequence? Theorem A solves the case when the support supp $\mu_{\gamma}$ is bounded 3 (compare [3, Lem. 3]), namely $\operatorname{supp} \mu_{\gamma} \subset[0,1 / Q(\gamma)]$. The goal of the present paper is to extend the method used in $\left[3\right.$ to include measures $\mu_{\gamma}$ in (1.3) having unbounded support, namely to certain cases with $Q(\gamma)=0$ or, equivalently, to cases where $\Gamma_{\gamma}$ has no positive radius of convergence.

We will see that Karlin's problem can partially be reduced to Schoenberg's theorem about Pólya Frequency densities (PF-densities) (see [17, Ch. IV], 6]). Our main result is the following theorem.

Theorem 1. Let $\gamma \in \mathcal{Z D}^{+}$be such that for any finite system of numbers $0<\beta_{1} \leq$ $\beta_{2} \leq \ldots \leq \beta_{m}<\infty, m \geq 1$, the sequence $\left\{\left(\beta_{1}^{n}+\beta_{2}^{n}+\ldots+\beta_{m}^{n}\right) \gamma_{n}\right\}_{n \geq 0}$ corresponds to a determinate Stieltjes moment problem. Then there exists $\Phi \in \mathcal{L} \mathcal{P}_{2}^{+}$such that $\gamma_{n}=\frac{\gamma_{0}}{\Phi(n)}, n \geq 0$.

Theorem 1 requires that the measure $d \mu(x):=d \mu_{\gamma}\left(x / \beta_{1}\right)+d \mu_{\gamma}\left(x / \beta_{2}\right)+\ldots+$ $d \mu_{\gamma}\left(x / \beta_{m}\right)$ should be the unique solution of the Stieltjes moment problem:

$$
\left(\beta_{1}^{n}+\beta_{2}^{n}+\ldots+\beta_{m}^{n}\right) \gamma_{n}=\int_{0}^{\infty} t^{n} d \mu(t), \quad n \geq 0 .
$$

It is worth mentioning that Theorem 1 applies to each $\gamma \in \mathcal{Z D}^{+}$satisfying the assumption of Theorem A; i.e. $\operatorname{supp} \mu_{\gamma}$ is bounded, because the moment problem on any bounded interval is indeed determinate (see, for example, [23]).

Two less general but probably more easily verified sufficient conditions for $\gamma \in$ $\mathcal{Z D}^{+}$to fulfill the assumptions of Theorem 1 are in the following corollary.

Corollary 1. Let $\gamma \in \mathcal{Z D}^{+}$satisfy one of the following properties:

A. There exists $a>0$ such that the sequence $\left\{\frac{\gamma_{n}}{n+a}\right\}_{n \geq 0}$ corresponds to a determinate Stieltjes moment problem.

B. The Carleman condition (see [11, 12]) holds: $\sum_{n \geq 1} \frac{1}{\gamma_{n}^{1 / 2 n}}=\infty$.

Then there exists $\Phi \in \mathcal{L P}_{2}^{+}$such that $\gamma_{n}=\frac{\gamma_{0}}{\Phi(n)}, n \geq 0$.

It is easy to see that any $\gamma \in \mathcal{Z D}^{+}$with $Q(\gamma)>0$ satisfies both conditions, A and $\mathrm{B}$, of Corollary 1 .

Before we turn to the proofs of our results we should like to make the following general remarks on zero-diminishing sequences:

1) In 1951, Schoenberg [27] proved that for every $\gamma \in \mathcal{Z D}^{+}$of Laguerre type, except for $\gamma=\left\{\gamma_{0} \cdot e^{b n}\right\}_{n \geq 0}, b \in \mathbb{R}$, there exists a representing measure $\mu_{\gamma}$ in (1.3) of the form $d \mu_{\gamma}(x)=\left(\gamma_{0} / x\right) f(\log 1 / x) d x$, where $f(x)$ is a PF-density (see [20, p. 31]).

2) In 1993, the following related problem was solved in [5]: to characterize all real sequences $\gamma$ for which

$$
\mathbb{Z}_{[0, A]}\left(T_{\gamma} p\right) \leq \mathbb{Z}_{[0, A]}(p) \text { for all } A>0, p \in \mathcal{P} .
$$

\footnotetext{
${ }^{3} \operatorname{supp} \mu:=\{x \in \mathbb{R} \mid \forall \varepsilon>0: \mu((x-\varepsilon, x+\varepsilon))>0\}$.
} 
The paper [3] started out from this work.

3) Note that for $\gamma \in \mathcal{Z D}^{+}$the reciprocal sequence $\tau:=\left\{\tau_{n}\right\}_{n \geq 0}$, with $\tau_{n}:=$ $1 / \gamma_{n}, n \geq 0$, has an inverse property:

$$
\mathbb{Z}_{\mathbb{R}}\left(T_{\tau} p\right) \geq \mathbb{Z}_{\mathbb{R}}(p), \quad p \in \mathcal{P} .
$$

4) In 1914, Pólya and Schur 24 solved a problem related to (1.8): they characterized all sequences $\tau$ of real numbers so that the transformation $T_{\tau}$ maps real polynomials with only real zeros to polynomials of the same type.

5) Other questions related to Karlin's problem have been discussed in A. Iserles, S.P. Norsett and E.B. Saff [19], T. Craven and G. Csordas [16] and Bakan, Craven and Csordas 4 .

\section{Homogeneously determinate measures on the positive half-Line}

In the sequel, we write $\mathcal{M}^{*}\left(\mathbb{R}^{+}\right)$and $\mathcal{M}^{*}(\mathbb{R})$ for the sets of all non-negative Borel measures on $\mathbb{R}$ with all moments finite and with non-empty supp $\mu$ contained in $\mathbb{R}^{+}$or $\mathbb{R}$, respectively. We say that $\mu \in \mathcal{M}^{*}(\mathbb{R})$ is determinate in the sense of Hamburger (in short: $\mu \in \operatorname{det} \mathcal{H}$ ) if $\mu$ is the only measure in $\mathcal{M}^{*}(\mathbb{R})$ with the same moments as $\mu$, and we say that $\mu$ is indeterminate in the sense of Hamburger (in short: $\mu \in \operatorname{indet} \mathcal{H}$ ) if there exist different measures in $\mathcal{M}^{*}(\mathbb{R})$ with the same moments as $\mu$. Replacing in the above sentence $\mathcal{M}^{*}(\mathbb{R})$ by $\mathcal{M}^{*}\left(\mathbb{R}^{+}\right)$we get a definition of the sets $\operatorname{det} \mathcal{S}$ and indet $\mathcal{S}$ of measures which are called determinate and indeterminate in the sense of Stieltjes, correspondingly.

The index of determinacy of $\mu \in \operatorname{det} \mathcal{H}$ is defined as

$$
\operatorname{ind}(\mu):=\sup \left\{k \in \mathbb{N}_{0} \mid \mu_{k} \in \operatorname{det} \mathcal{H}\right\},
$$

where $\mathbb{N}_{0}:=\{0,1,2, \ldots\}$ and $d \mu_{s}(x):=\left(x^{2}+1\right)^{s} d \mu(x), s \in \mathbb{R}$. Note that $\operatorname{ind}(\mu)$ coincides with the index $\operatorname{ind}_{i} \mu$ introduced in [8, p. 2795].

Let

$$
\pi_{\infty}:=\left\{\left(\beta_{1}, \beta_{2}, \ldots, \beta_{n}\right) \mid 0<\beta_{1} \leq \beta_{2} \leq \ldots \leq \beta_{n}<\infty, 1 \leq n<\infty\right\} .
$$

For arbitrary $\mu \in \mathcal{M}^{*}\left(\mathbb{R}^{+}\right)$and $\beta:=\left(\beta_{1}, \beta_{2}, \ldots, \beta_{n}\right) \in \pi_{\infty}$ let $\Gamma_{\beta} \mu$ be the measure

$$
\Gamma_{\beta} \mu(A):=\sum_{k=1}^{n} \mu\left(A / \beta_{k}\right), \quad A \in \mathcal{B}(\mathbb{R}),
$$

where $\mathcal{B}(\mathbb{R})$ are the Borel sets in $\mathbb{R}$. The measures in

$$
\operatorname{det}_{\mathrm{h}} \mathcal{S}:=\left\{\mu \in \mathcal{M}^{*}\left(\mathbb{R}^{+}\right) \mid \Gamma_{\beta} \mu \in \operatorname{det} \mathcal{S} \text { for all } \beta \in \pi_{\infty}\right\}
$$

are related to the assumptions in Theorem 1 and are called homogeneously determinate on $\mathbb{R}^{+}$. Note that

$$
\mu \in \operatorname{det}_{\mathrm{h}} \mathcal{S}, \beta \in \pi_{\infty} \Rightarrow \Gamma_{\beta} \mu \in \operatorname{det}_{\mathrm{h}} \mathcal{S} .
$$

Lemma 2.1. If $\mu \in \operatorname{det}_{h} \mathcal{S}$, then for arbitrary $\beta \in \pi_{\infty}$ and $1 \leq p<\infty$ the set $\mathcal{P}$ is dense in the space $L_{2}\left(\mathbb{R},\left(1+x^{2}\right)^{p} d \Gamma_{\beta} \mu\right)$.

Proof. First recall M. Riesz's theorem [25, which implies that $\mathcal{P}$ is dense in $L_{2}\left(\mathbb{R},\left(1+x^{2}\right) d \mu\right)$ iff $\mu \in \operatorname{det} \mathcal{H}$. If $\mu \in \operatorname{indet} \mathcal{H}$ and $\mathcal{P}$ is dense in $L_{2}(\mathbb{R}, d \mu)$, then $\mu$ is called a Nevanlinna extremal measure ( $N$-extremal in short). All $N$-extremal measures are discrete and satisfy: $\sum_{\lambda \in \operatorname{supp} \mu}\left(1+\lambda^{2}\right)^{-1}<\infty$ (cf. [1, p. 100]). By (2.2) it suffices to prove polynomial denseness in $L_{2}\left(\mathbb{R},\left(1+x^{2}\right)^{p} d \mu(x)\right)$ for arbitrary 
$\mu \in \operatorname{det}_{\mathrm{h}} \mathcal{S}$. However, for such $\mu$, [7, Cor. 1.2] implies that $\mathcal{P}$ is dense in $L_{2}(\mathbb{R}, d \mu)$ and then, by M. Riesz's theorem: $\mu_{-1} \in \operatorname{det} \mathcal{H}$.

Assume that $l:=$ ind $\left(\mu_{-1}\right)<\infty$. Then Lemma 3.7 and Theorem 3.9 in 8 ] ensure the existence of an $N$-extremal measure $\sigma$ with $d \sigma(x)=\sum_{\lambda \in \Lambda} \sigma_{\lambda} \delta(x-\lambda)$ (here $\delta(x)$ denotes the Dirac generalized function), and a subset $\Lambda_{0} \subset \Lambda$ with $l+1$ distinct elements such that

$$
\frac{d \mu(x)}{1+x^{2}}=d \sigma(x)-\sum_{\lambda \in \Lambda_{0}} \sigma_{\lambda} \delta(x-\lambda)=\sum_{\lambda \in \Lambda \backslash \Lambda_{0}} \sigma_{\lambda} \delta(x-\lambda) .
$$

Next we choose $\beta_{*} \in(1,+\infty) \backslash\left\{\lambda / \lambda_{1} \mid \lambda \in \Lambda, \lambda_{1} \in \Lambda \backslash\left(\{0\} \cup \Lambda_{0}\right)\right\}$ and write $\left\{\beta_{*} \cdot a_{k}\right\}_{k \geq 0}$ for the sequence of distinct points in the infinite set $\left(\beta_{*} \cdot\left(\Lambda \backslash \Lambda_{0}\right)\right) \backslash \Lambda$. Look at the measure

$$
d \nu(x):=\frac{d \mu(x)}{1+x^{2}}+\frac{d \mu\left(x / \beta_{*}\right)}{1+\left(x / \beta_{*}\right)^{2}}=\sum_{\lambda \in \Lambda \backslash \Lambda_{0}} \sigma_{\lambda} \delta(x-\lambda)+\sum_{\lambda \in \Lambda \backslash \Lambda_{0}} \sigma_{\lambda} \delta\left(x-\beta_{*} \lambda\right) .
$$

According to Lemma D in [8] the measure

$$
d \omega(x):=\sum_{\lambda \in \Lambda \backslash \Lambda_{0}} \sigma_{\lambda} \delta(x-\lambda)+\sum_{k=0}^{l} \sigma_{a_{k}} \delta\left(x-a_{k} \beta_{*}\right)
$$

is $N$-extremal and so $\mathcal{P}$ is not dense in $L_{2}\left(\mathbb{R},\left(1+x^{2}\right) d \omega(x)\right)$. Consequently (see (2.3) ) $\mathcal{P}$ is not dense in $L_{2}\left(\mathbb{R}, d \Gamma_{\beta} \mu(x)\right)$ as well, for $\beta:=\left(1, \beta_{*}\right)$. Corollary 1.2 in [7, p. 211] then gives $\Gamma_{\beta} \mu \in$ indet $\mathcal{S}$, which contradicts (2.2) and completes the proof of Lemma 2.1.

It should be noted that there exist measures $\mu \in \operatorname{det} \mathcal{S} \backslash \operatorname{det}_{\mathrm{h}} \mathcal{S}$ such that $\operatorname{ind}(\mu):=+\infty$. Indeed, for $d \mu(x)=\sum_{k>1} e^{-1,5 k^{2}} \delta\left(x-e^{k}\right)$ it follows from 77. Th. 3.8], 22, Th. 17] and [2, Cor. 2.1] that $\mu \in \operatorname{det} \mathcal{S}$, $\operatorname{ind}(\mu):=+\infty$ but $\Gamma_{(0.75,1)} \mu \notin \operatorname{det} \mathcal{S}$. We omit the details.

Let $\mu \diamond \nu$ denote the convolution of $\mu, \nu \in \mathcal{M}^{*}\left(\mathbb{R}^{+}\right)$defined as

$$
(\mu \diamond \nu)(A)=\iint_{\mathbb{R}^{2}} \chi_{A}(x \cdot y) d \mu(x) d \nu(y), A \in \mathcal{B}(\mathbb{R}),
$$

where $\chi_{A}$ denotes the characteristic function of the set $A$. Properties of this convolution can be found in [10, Ch. VIII, §1] and 9, p. 244]. In particular, if $s_{\xi}(\mu):=\int_{\mathbb{R}^{+}} x^{\xi} d \mu(x)$, for some $\xi$ with $\operatorname{Re} \xi \geq 0$, then $s_{\xi}(\mu \diamond \nu)=s_{\xi}(\mu) \cdot s_{\xi}(\nu)$.

Let $\delta_{a}$ denote the Dirac measure at $a \in \mathbb{R}$, i.e. $d \delta_{a}(x):=\delta(x-a)$. Then for any $A \in \mathcal{B}(\mathbb{R})$ and $a>0$ we have $\left(\delta_{a} \diamond \mu\right)(A)=\mu(A / a)$. Thus, for $\beta \in \pi_{\infty}$ and $\mu, \nu \in \mathcal{M}^{*}\left(\mathbb{R}^{+}\right)$, one obtains

$$
\Gamma_{\beta} \mu=\left(\delta_{\beta_{1}}+\delta_{\beta_{2}}+\ldots+\delta_{\beta_{n}}\right) \diamond \mu, \quad \Gamma_{\beta}(\mu \diamond \nu)=\left(\Gamma_{\beta} \mu\right) \diamond \nu=\mu \diamond\left(\Gamma_{\beta} \nu\right) .
$$

Lemma 2.2. Let $\mu, \nu \in \mathcal{M}^{*}\left(\mathbb{R}^{+}\right)$and assume that for arbitrary $\theta \in(0,1]$ there exists a finite positive constant $C_{\theta}$ such that $\nu(A / \theta) \leq C_{\theta} \cdot \nu(A)$ holds for any $A \in \mathcal{B}(\mathbb{R})$ (in short: $\delta_{\theta} \diamond \nu \leq C_{\theta} \cdot \nu$ ). Then $\mu \diamond \nu \in \operatorname{det} \mathcal{S}$ implies $\mu \in \operatorname{det}_{\mathrm{h}} \mathcal{S}$.

Proof. Lemma 2.2 and Remark 2.3 in [9, pp. 246, 247] state that for any $\rho, \omega \in$ $\mathcal{M}^{*}\left(\mathbb{R}^{+}\right)$, with $\omega$ not a positive multiple of $\delta_{0}$, one has

$$
\rho \diamond \omega \in \operatorname{det} \mathcal{S} \Rightarrow \rho \in \operatorname{det} \mathcal{S} .
$$


For $a>0$ use $\rho=\mu \diamond\left(\delta_{a} \diamond \nu\right), \omega=\delta_{1 / a}$ in (2.6) and apply the relation $\delta_{a} \diamond \delta_{b}=\delta_{a \cdot b}$, $a, b>0$, to obtain $\rho \diamond \omega=\mu \diamond \nu$ and

$$
\mu \diamond\left(\delta_{a} \diamond \nu\right) \in \operatorname{det} \mathcal{S} .
$$

Moreover, our assumption concerning $\nu$ applied to $A=B / b$ and $\theta=a / b \in(0,1]$ gives

$$
\delta_{a} \diamond \nu \leq C_{a / b} \cdot\left(\delta_{b} \diamond \nu\right), \quad 0<a \leq b<\infty .
$$

It now follows from (2.8) and (2.5) that for arbitrary $\beta \in \pi_{\infty}$ we have

$$
\Gamma_{\beta} \nu \leq C_{\beta} \cdot\left(\delta_{\beta_{n}} \diamond \nu\right), \text { where } C_{\beta}:=\left(C_{\beta_{1} / \beta_{n}}+\ldots+C_{\beta_{n-1} / \beta_{n}}+C_{1}\right),
$$

and therefore, by (2.4) and (2.5), $\left(\Gamma_{\beta} \mu\right) \diamond \nu=\mu \diamond\left(\Gamma_{\beta} \nu\right) \leq C_{\beta} \cdot\left(\mu \diamond\left(\delta_{\beta_{n}} \diamond \nu\right)\right)$. From (2.7) we deduce $\mu \diamond\left(\delta_{\beta_{n}} \diamond \nu\right) \in \operatorname{det} \mathcal{S}$ so that [7, Th. 3.9] gives $\left(\Gamma_{\beta} \mu\right) \diamond \nu \in \operatorname{det} \mathcal{S}$. Our conditions imposed on $\nu$ do not allow it to be a Dirac measure. Thus (2.6) yields $\Gamma_{\beta} \mu \in \operatorname{det} \mathcal{S}$ for every $\beta \in \pi_{\infty}$, which is our claim.

Let $\mathfrak{P}\left(\mathbb{R}^{+}\right)$be the class of all continuous and non-negative functions $g$ on $(0,+\infty)$ which, at infinity, do not grow faster than some monomial $x^{q}, q \geq 0$, and have at most finitely many positive zeros. Let

$$
\mathbb{A}:=\left\{ \pm g(x) \cdot \prod_{k=1}^{m}\left(x-x_{k}\right) \mid 0<x_{1}<x_{2}<\ldots<x_{m}<\infty, m \in \mathbb{N}_{0}, g \in \mathfrak{P}\left(\mathbb{R}^{+}\right)\right\}
$$

where $\prod_{k=1}^{0}:=1$. The following is an immediate consequence of Lemma 2.1

Lemma 2.3. Let $\mu \in \operatorname{det}_{\mathrm{h}} \mathcal{S}, f(x)=g(x) \cdot \prod_{k=1}^{m}\left(x-x_{k}\right) \in \mathbb{A}$, where $g \in \mathfrak{P}\left(\mathbb{R}^{+}\right)$ belongs to $\bigcap_{\lambda>0} L_{1}(\mathbb{R}, d \mu(x / \lambda))$. Then for any finite system $0<y_{1}<y_{2}<\ldots<$ $y_{n}<\infty$, there exists a polynomial sequence $P_{j}(x)=\left(1 / j+Q_{j}(x)^{2}\right) \cdot \prod_{k=1}^{m}\left(x-x_{k}\right)$ for $j \geq 1$, with $Q_{j} \in \mathcal{P}$, which satisfies

$$
\lim _{j \rightarrow \infty} \int_{0}^{\infty} P_{j}\left(t y_{k}\right) d \mu(t)=\int_{0}^{\infty} f\left(t y_{k}\right) d \mu(t), \quad 1 \leq k \leq n .
$$

Proof. If $g \in \mathfrak{P}\left(\mathbb{R}^{+}\right)$, then $(1+x)^{r} g \in L_{1}(\mathbb{R}, d \mu(x / \lambda))$ for arbitrary $r, \lambda>0$, so that, according to Lemma 2.1. there exists a polynomial sequence $\left\{Q_{j}\right\}_{j \geq 1}$ which approximates $\sqrt{g(x)}$ in the space $L_{2}\left(\mathbb{R},(1+x)^{m} d \nu(x)\right)$, with $\nu:=\Gamma_{\beta} \mu, \beta:=$ $\left(y_{1}, y_{2}, \ldots, y_{n}\right)$. Then, with $A:=\prod_{k=1}^{m}\left(1+x_{k}\right)$ we obtain

$$
\begin{aligned}
& \frac{1}{A} \int_{0}^{\infty}\left|g(t) \prod_{k=1}^{m}\left(t-x_{k}\right)-Q_{j}(t)^{2} \prod_{k=1}^{m}\left(t-x_{k}\right)\right| d \nu(t) \leq \int_{0}^{\infty}\left|g(t)-Q_{j}(t)^{2}\right|(1+t)^{m} d \nu(t) \\
& \leq\left(\int_{0}^{\infty}\left|\sqrt{g(t)}-Q_{j}(t)\right|^{2}(1+t)^{m} d \nu(t) \cdot \int_{0}^{\infty}\left|\sqrt{g(t)}+Q_{j}(t)\right|^{2}(1+t)^{m} d \nu(t)\right)^{1 / 2} \rightarrow 0,
\end{aligned}
$$

and

$$
(1 / j) \cdot \int_{0}^{\infty} \prod_{k=1}^{m}\left|t-x_{k}\right| d \nu(t) \leq(A / j) \cdot \int_{0}^{\infty}(1+t)^{m} d \nu(t) \rightarrow 0, \text { as } j \rightarrow \infty .
$$

This implies (2.10). 


\section{Auxiliary lemmas}

Let $\mathcal{Z D}_{\infty}^{+}$be the subset of $\gamma \in \mathcal{Z D}^{+}$with $Q(\gamma)=0$. We need to prove Theorem 1 only for $\gamma \in \mathcal{Z D}_{\infty}^{+}$with $\mu_{\gamma} \in \operatorname{det}_{\mathrm{h}} \mathcal{S}$ (see (1.3)). For such $\gamma$ the transformation $T_{\gamma}$ can be written as

$$
T_{\gamma} p(x)=\int_{0}^{\infty} p(x t) d \mu_{\gamma}(t), \quad p \in \mathcal{P} .
$$

We can extend the domain of definition of $T_{\gamma}$ to

$$
f \in \boldsymbol{O}_{\gamma}:=\boldsymbol{O} \cap\left(\bigcap_{\lambda>0} L_{1}\left(\mathbb{R}, d \mu_{\gamma}(x / \lambda)\right)\right)
$$

by

$$
T_{\gamma} f(x):=\int_{0}^{\infty} f(x t) d \mu_{\gamma}(t), \quad x>0 .
$$

For arbitrary $A \subset \mathbb{R}$ and $g: A \mapsto \mathbb{R}$ let $S_{A}(g)$ denote the number of sign changes of $g$ on $A$. More precisely (see [17, Ch. IV, $\S 1]$ ),

$$
S_{A}(g):=\sup \left\{n \geq 1 \mid \exists\left\{t_{i}\right\}_{i=0}^{n} \subset A, t_{0}<t_{1}<\ldots<t_{n}: g\left(t_{j}\right) g\left(t_{j+1}\right)<0,0 \leq j \leq n-1\right\}
$$

with $\sup \emptyset:=0$ and $S_{+}(g):=S_{(0,+\infty)}(g)$. Observe that the definition of $\mathfrak{P}\left(\mathbb{R}^{+}\right)$ implies that the number of sign changes on $(0,+\infty)$ of a function of the form (2.9) is exactly $m$. An obvious application of Lemma 2.3 then gives

Lemma 3.1. Let $\gamma \in \mathcal{Z D}_{\infty}^{+}, \mu_{\gamma} \in \operatorname{det}_{\mathrm{h}} \mathcal{S}$ and $f \in \mathbb{O}_{\gamma}$. Then

$$
S_{+}\left(T_{\gamma} f\right) \leq S_{+}(f) \text {. }
$$

Proof. Assume there exists $f \in \mathbb{\Pi}_{\gamma}$ satisfying $r:=S_{+}\left(T_{\gamma} f\right)>S_{+}(f)=: m \geq$ 0 . Since $S_{A}(g) \equiv S_{A}(-g)$ we can assume that $f$ in (2.9) has a form: $f(x)=$ $g(x) \prod_{k=1}^{m}\left(x-x_{k}\right)$, where $g \in \mathfrak{P}\left(\mathbb{R}^{+}\right) \cap \bigcap_{\lambda>0} L_{1}(\mathbb{R}, d \mu(x / \lambda))$. Choose points $0<$ $y_{0}<y_{2}<\ldots<y_{r}<\infty$ such that

$$
T_{\gamma} f\left(y_{i}\right) \cdot T_{\gamma} f\left(y_{i+1}\right)<0,0 \leq i \leq r-1 .
$$

If we approximate $f$ by the polynomial sequence $\left\{P_{j}\right\}_{j \geq 1}$ from Lemma 2.3, then by construction $\mathbb{Z}_{\mathbb{R}}\left(P_{j}\right)=m, j \geq 1$, and for sufficiently large $j$,

$$
T_{\gamma} P_{j}\left(y_{i}\right) \cdot T_{\gamma} P_{j}\left(y_{i+1}\right)<0, \quad 0 \leq i \leq r-1,
$$

i.e. $\mathbb{Z}_{\mathbb{R}}\left(T_{\gamma} P_{j}\right) \geq r>m$, which contradicts $\gamma \in \mathcal{Z D}_{\infty}^{+}$.

Lemma 3.2. Let $\gamma \in \mathcal{Z D}_{\infty}^{+}$and $\mu_{\gamma} \in \operatorname{det}_{\mathrm{h}} \mathcal{S}$. Then $\mu_{\gamma}(\{0\})=0$.

Proof. Assume that $a:=\mu_{\gamma}(\{0\})>0$. Then

$$
d \mu_{\gamma}(x)=a \cdot \delta(x)+d \nu_{\gamma}(x) ; \quad \nu_{\gamma} \in \mathcal{M}^{*}\left(\mathbb{R}^{+}\right) ; \quad \nu_{\gamma}([0, \varepsilon]) \rightarrow 0, \varepsilon \downarrow 0 .
$$

Choose $0<\alpha_{1}<\alpha_{2}<1$ and let $p(x)=\left(x-\alpha_{1}\right)\left(x-\alpha_{2}\right)$. Then the polynomial $q(x)$ with $T_{\gamma} q(x)=p(x)$ satisfies $\lim _{x \rightarrow+\infty} q(x)=+\infty$ and therefore one can choose a finite positive number $m>-\min _{x \in \mathbb{R}^{+}} q(x)$. For every $\varepsilon>0$ let

$$
f_{q}^{\varepsilon}(x):=(m+q(x)) \cdot \chi_{(\varepsilon,+\infty)}(x)+\left(-A+\frac{x}{\varepsilon}(A+q(\varepsilon)+m)\right) \cdot \chi_{[0, \varepsilon]}(x),
$$


where $A:=m \cdot\left(\gamma_{0}-a\right) / a-q(0)$. Clearly $f_{q}^{\varepsilon} \in C\left(\mathbb{R}^{+}\right)$, positive for $x \geq \varepsilon$ and linear on $[0, \varepsilon]$. It follows that $f_{q}^{\varepsilon}$ can vanish at most once in the interval $(0, \varepsilon)$. Therefore $f_{q}^{\varepsilon} \in \mathbb{Q}$ and Lemma 3.1 applies to give

$$
S_{+}\left(T_{\gamma} f_{q}^{\varepsilon}\right) \leq S_{+}\left(f_{q}^{\varepsilon}\right) \leq 1, \quad \varepsilon>0
$$

Then for any $x>0$,

$$
\begin{aligned}
T_{\gamma} f_{q}^{\varepsilon}(x) & =\int_{0}^{\infty} f_{q}^{\varepsilon}(x t) d \mu_{\gamma}(t) \\
& =-a \cdot A+\int_{0}^{\infty}(m+q(t)) d \nu_{\gamma}(t / x)+\int_{0}^{\varepsilon}\left[f_{q}^{\varepsilon}(t)-q(t)-m\right] d \nu_{\gamma}(t / x)
\end{aligned}
$$

hence,

$$
\left.T_{\gamma} f_{q}^{\varepsilon}(x)=p(x)-m \cdot \nu_{\gamma}([0, \varepsilon / x])\right)+\int_{0}^{\varepsilon}\left[f_{q}^{\varepsilon}(t)-q(t)\right] d \nu_{\gamma}(t / x), \quad \varepsilon>0 .
$$

The function $f_{q}^{\varepsilon}(t)-q(t)$ is uniformly bounded on $0 \leq t \leq \varepsilon$, so that (3.4) and (3.6) imply $\lim _{\varepsilon \downarrow 0} T_{\gamma} f_{q}^{\varepsilon}(x)=p(x)$ uniformly over $x \geq \delta$ for every $\delta>0$. Thus, for sufficiently small $\varepsilon>0: S_{+}\left(T_{\gamma} f_{q}^{\varepsilon}\right) \geq S_{+}(p)=2$, a contradiction to (3.5).

\section{Proof of Theorem 1}

We first study the shifted sequence $\omega:=\left\{\gamma_{1}, \gamma_{2}, \ldots, \gamma_{n}, \ldots\right\}$, which also belongs to $\mathcal{Z D}_{\infty}^{+}$(see remark made before (1.4) ) and corresponds to the measure $\mu_{\omega}$ given by $d \mu_{\omega}=x \cdot d \mu_{\gamma}(x)$. Since the product of a finite number of functions in 0 belongs to $\boldsymbol{Z}$ and $\log x-\log a=(x-a) \cdot \frac{\log x-\log a}{x-a} \in \boldsymbol{A}$ for $x, a>0$, we have

$$
\{p(\log x) \mid p \in \mathcal{P}\} \subset \boldsymbol{\nearrow}_{\omega}=\boldsymbol{O} \cap\left(\bigcap_{\lambda>0} L_{1}\left(\mathbb{R}, d \mu_{\omega}(x / \lambda)\right)\right) .
$$

In (3.2) we set $x=e^{u}, t=e^{-v}$, and write $F(u):=f\left(e^{u}\right), V_{\omega} F(u):=T_{\omega} f\left(e^{u}\right)$ and $d L(v):=-d \mu_{\omega}\left(e^{-v}\right)$. Then (3.2) becomes

$$
V_{\omega} F(u)=\int_{-\infty}^{+\infty} F(u-v) d L(v)
$$

where $L$ can be looked at as the function $L(x)=\mu_{\omega}\left(\left[e^{-x},+\infty\right)\right), x \in \mathbb{R}$. Now Lemma 3.1 and (4.1) give

$$
S_{\mathbb{R}}\left(V_{\omega} p\right) \leq S_{\mathbb{R}}(p), \quad p \in \mathcal{P} .
$$

Recalling the proof of [17, Th. 4.1] we note that essentially nothing changes if, in that proof, we replace the convolution of $\pi^{-1 / 2} e^{-t^{2}}$ with the variation-diminishing frequency function $\varphi$ by

$$
\alpha(x):=\frac{1}{\sqrt{\pi}} \int_{\mathbb{R}} e^{-(x-t)^{2}} d L(t) .
$$

Indeed, the two-sided Laplace transform of $\alpha(x)$,

$$
\int_{\mathbb{R}} e^{-s x} \alpha(x) d x=e^{\frac{s^{2}}{4}} \int_{\mathbb{R}} e^{-s x} d L(x)=e^{\frac{s^{2}}{4}} \int_{0}^{\infty} x^{s+1} d \mu_{\gamma}(x),
$$

is analytic in the domain $\operatorname{Re} s>-1$ and (4.3) gives

$$
S_{\mathbb{R}}\left(\int_{\mathbb{R}} \alpha(x-t) p(t) d t\right) \leq S_{\mathbb{R}}(p), \quad p \in \mathcal{P} .
$$


This establishes the existence of

$$
E(s)=E(0) e^{-a s^{2}+b s} \prod_{k \geq 1}^{m}\left(1+\frac{s}{\alpha_{k}}\right) e^{-\frac{s}{\alpha_{k}}},
$$

where $a \geq 0, b \in \mathbb{R}, \alpha_{k} \geq 1, k \geq 1, m \in \mathbb{N} \cup\{\infty\}$ and $\sum_{k \geq 1} 1 / \alpha_{k}^{2}<\infty$, such that:

$$
\int_{\mathbb{R}} e^{-s x} d L(x)=\int_{0}^{\infty} x^{s+1} d \mu_{\gamma}(x)=\frac{1}{E(s)},
$$

for $\operatorname{Re} s>-1$. Lemma 3.2 allows us to pass to the limit $\operatorname{Re} s \rightarrow-1$ in the second equation of (4.4) so that (4.4) holds indeed for all $\operatorname{Re} s \geq-1$ and consequently $\alpha_{k}>1$ for all $k \geq 1$. Therefore (4.4) is actually the required representation for $\gamma=\left\{\gamma_{0}, \gamma_{1}, \ldots\right\}$ if we set $\Phi(s):=E(s-1)$.

\section{Proof of Corollary 1}

5.1. Proof for condition A. For any sequence $\gamma$ fulfilling condition A we show that the corresponding measure $\mu_{\gamma}$ (see (1.3) ) belongs to $\operatorname{det}_{\mathrm{h}} \mathcal{S}$; i.e. $\gamma$ satisfies the assumptions of Theorem 1. For arbitrary $a>0$ let $\tau_{a} \in \mathcal{M}^{*}\left(\mathbb{R}^{+}\right)$be defined as follows: $d \tau_{a}(x):=x^{a-1} \cdot \chi_{[0,1]}(x) d x$. Since $s_{n}\left(\tau_{a}\right)=1 /(n+a)$ we find $s_{n}\left(\mu_{\gamma} \diamond \tau_{a}\right)=$ $\gamma_{n} /(n+a), n \in \mathbb{N}_{0}$, from which by condition $\mathrm{A}$ it follows that

$$
\mu_{\gamma} \diamond \tau_{a} \in \operatorname{det} \mathcal{S} .
$$

But for arbitrary $A \in \mathcal{B}(\mathbb{R})$ and $\theta \in(0,1]$,

$$
\tau_{a}(A / \theta)=\int_{A / \theta} x^{a-1} \cdot \chi_{[0,1]}(x) d x=\frac{1}{\theta^{a}} \cdot \int_{A} x^{a-1} \cdot \chi_{[0, \theta]}(x) d x \leq \frac{1}{\theta^{a}} \cdot \tau_{a}(A),
$$

which shows that the measure $\tau_{a}$ satisfies the condition of Lemma 2.2. Therefore (5.1) and Lemma 2.2 imply $\mu_{\gamma} \in \operatorname{det}_{\mathrm{h}} \mathcal{S}$.

5.2. Proof for condition B. Since $\lim _{n \rightarrow \infty} \gamma_{n}=\infty$ for every $\gamma \in \mathcal{Z D}_{\infty}^{+}$the assumption implies $\sum_{n \geq 1}\left(\gamma_{n} /(n+a)\right)^{-1 /(2 n)}=\infty, \quad a>0$, so that Carleman's theorem [28, Th. 1.11] gives $\left\{\gamma_{n} /(n+a)\right\}_{n>0} \in \operatorname{det} \mathcal{S}, a>0$. Condition A in Corollary 1 is therefore fulfilled, and this completes the proof of part B.

\section{ACKNOWLEDGMENT}

The authors thank the referee for important remarks resulting in considerable modifications of Lemma 2.2 and the proof for condition A in Corollary 1.

\section{REFERENCES}

1. N.I. Akhiezer, The classical moment problem, Oliver and Boyd, Edinburgh, 1965.

2. A. Bakan, Polynomial density in $L_{p}(R, d \mu)$ and representation of all measures which generate a determinate Hamburger moment problem, Approximation, Optimization and Mathematical Economics (Pointe-à-Pitre, 1999), Physica, Heidelberg, 2001, 37-46. MR.1842874 (2002i:41004)

3. A. Bakan, T. Craven, G. Csordas and A. Golub, Weakly increasing zero-diminishing sequences, Serdica Math. J. 22 (1996), 4, 547-570. MR.1483605 (98k:26025)

4. A. Bakan, T. Craven and G. Csordas, Interpolation and the Laguerre-Pólya class, Southwest J. Pure Appl. Math. 1 (2001), 38-53. Available at rattler.cameron.edu/swjpam/swjpam.html. MR 1841319 (2002g:41001)

5. A. Bakan and A. Golub, Some negative results on sequences of multipliers of the first kind, Ukrain. Mat. Zh. 44 (1992), 3, 305-309 (Russian); English transl.: Ukrainian Math. J. 44 (1992), 3, 264-268. MR1185923 (93m:26030) 
6. A. Bakan and St. Ruscheweyh, On the existence of generalized Pólya frequency functions corresponding to entire functions with zeros in angular sectors, In honor of Professor Erwin Kreyszig on the occasion of his 80th birthday, Complex Var. Theory Appl. 47 (2002), 7, 565-576. MR1918559 (2003g:42012)

7. Ch. Berg and M. Thill, Rotation invariant moment problems, Acta Math. 167 (1991), 207-227. MR.1120603 (92j:44004)

8. Ch. Berg and A. Duran, The index of determinacy for measures and the $l^{2}$-norm of orthonormal polynomials, Trans. Amer. Math. Soc. 347 (1995), 2795-2811. MR1308001 (96f:30033)

9. Ch. Berg and A. Duran, A transformation from Hausdorff to Stieltjes moment sequences, Ark. Mat. 42 (2004), 239-257. MR2101386 (2005j:40006)

10. N. Bourbaki, Intégration, Chapitres VI-VIII, Hermann et Cie, Paris 1244 (1956), 1281 (1959), 1306 (1963). MR0124722 (23:A2033). MR0179291 (31:3539) MR2098271 (2005f:28001)

11. T. Carleman, Sur le problème des moments, Comptes Rendus 174 (1922), 1680-1682.

12. T. Carleman, Les fonctions quasi-analytiques, Gauthier-Villars, Paris, 1926, 115 pp.

13. T. Craven and G. Csordas, Jensen polynomials and the Turán and Laguerre inequalities, Pacific. J. Math. 136 (1989), 241-260. MR.978613 (90a:26035)

14. T. Craven and G. Csordas, Zero-diminishing linear transformations, Proc. Amer. Math. Soc. 80 (1980), 4, 544-546. MR587923 (82a:12013)

15. T. Craven and G. Csordas, Complex zero decreasing sequences, Methods Appl. Anal. 2 (1995), 4, 420-441. MR.1376305 (98a:26015)

16. T. Craven and G. Csordas, Problems and theorems in the theory of multiplier sequences, Serdica Math. J. 22 (1996), 4, 515-524. MR1483603 (98k:26024)

17. I. Hirschman and D. Widder, The convolution transform, Princeton Univ. Press, Princeton, New Jersey, 1955. MR0073746 (17:479c)

18. L. Iliev, Laguerre entire functions, Publ. House of the Bulg. Acad. Sci., Sofia, 1987, 188 pp. MR 937705 (89h:30042)

19. A. Iserles, S.P. Norsett and E. B. Saff, On transformations and zeros of polynomials, Rocky Mountain J. Math. 21 (1991), 331-357. MR1113932 (92f:42028)

20. S. Karlin, Total Positivity, vol. 1. Stanford Univ. Press, Stanford, Calif., 1968. MR0230102 $(37: 5667)$

21. E. Laguerre, Sur quelques points de la théorie des équations numériques, Acta Math. 4 (1884), 97-120. MR 1554633

22. S. N. Mergelyan, Weighted approximation by polynomials, Uspekhi Mat. Nauk 11 (1956), 107-152 (Russian); English transl.: Amer. Math. Soc. Transl. 10 (1958), 59-106. MR0094633 (20:1146)

23. I. P. Natanson, Constructive Function Theory, Vol. II, Approximation in Mean (translation by J. R. Schulenberger), Frederick Ungar Pub. Co., New York, 1965. MR0196341 (33:4529b)

24. G. Pólya and J. Schur, Über zwei Arten von Faktorenfolgen in der Theorie der algebraischen Gleichungen, J. Reine Angew. Math. 144 (1914), 89-113.

25. M. Riesz, Sur le problème des moments et le théorème de Parseval correspondant, Acta Litt. Ac. Sci. Szeged 1 (1923), 209-225.

26. W. Rudin, Principles of mathematical analysis, 3rd. ed., McGraw-Hill, New York, 1976. MR0385023 (52:5893)

27. I. Schoenberg, On Pólya frequency functions I: The totally positive functions and their Laplace transforms, J. d'Anal. Math. 1 (1951), 331-374. MR0047732(13:923c)

28. J. Shohat and J. Tamarkin, The problem of moments, Math. Surveys, Number I, AMS, New York, 1943. MR0008438 (5:5c)

Institute of Mathematics, National Academy of Sciences of Ukraine, TereschenKivsKa Street 3, Kyiv 01601, Ukraine

E-mail address: andrew@bakan.kiev.ua

Mathematisches Institut, Universitat Würzburg, 97074 Würzburg, Germany

E-mail address: ruscheweyh@mathematik.uni-wuerzburg.de 